\title{
Smooth divisors of projective hypersurfaces
}

\author{
Philippe Ellia, Davide Franco and Laurent Gruson
}

\begin{abstract}
Let $X \subset \mathbb{P}^{n}$ be a smooth codimension 2 subvariety. We first prove a "positivity lemma" (Lemma 1.1) which is a direct application of the positivity of $N_{X}(-1)$. Then we first derive two consequences:

1) Roughly speaking the family of "biliaison classes" of smooth subvarieties of $\mathbb{P}^{5}$ lying on a hypersurface of degree $s$ is limited.

2) The family of smooth codimension 2 subvarieties of $\mathbb{P}^{6}$ lying on a hypersurface of degree $s$ is limited.

The result in 1) is not effective, but 2) is. Then we obtain precise inequalities connecting the usual numerical invariants of a smooth subcanonical subvariety $X \subset \mathbb{P}^{n}, n \geq 5$ (the degree $d$, the integer $e$ such that $\omega_{X} \simeq \mathcal{O}_{X}(e)$, the least degree, $s$, of a hypersurface containing $\left.X\right)$. In particular we prove: $s \geq n+1$ if $X$ is not a complete intersection.
\end{abstract}

Mathematics Subject Classification (2000). 14MO7, 14M1O, 14C20.

Keywords. Smooth codimension two subvarieties, projective space,complete intersections, positivity.

\section{Introduction}

We work over an algebraically closed field of arbitrary characteristic.

Ellingsrud-Peskine ([7]) proved that smooth surfaces in $\mathbb{P}^{4}$ are subject to strong limitations. Their whole argument is derived from the fact that the sectional genus of surfaces of degree $d$ lying on a hypersurface of degree $s$ varies in an interval of length $\frac{d(s-1)^{2}}{2 s}$. The aim of the present paper is to show that for smooth codimension two subvarieties of $\mathbb{P}^{n}, n \geq 5$, one can get a similar result with an interval whose length depends only on $s$. The main point is Lemma 1.1 whose proof is a direct application of the positivity of $N_{X}(-1)$ (where $N_{X}$ is the normal bundle of $X$ in $\mathbb{P}^{n}$ ). As a consequence of Lemma 1.1 (Remark 1.3) we get a series of $(n-3)$ inequalities the first one of which being Lemme 1 of [7]. The second (Theorem 1.4) was obtained in a preliminary version ([5]) by an essentially equivalent but more geometric argument.

Then we first derive two consequences: 
1) Roughly speaking (Theorem 2.1, Remark 2.5) the family of "biliaison classes" of smooth subvarieties of $\mathbb{P}^{5}$ lying on a hypersurface of degree $s$ is limited.

2) The family of smooth codimension two subvarieties of $\mathbb{P}^{6}$ lying on a hypersurface of degree $s$ is limited (Theorem 1.4).

The result quoted in 1) is not effective, but 2) is.

In the last section we try to obtain precise inequalities connecting the usual numerical invariants of a smooth subcanonical subvariety $X$ of $\mathbb{P}^{n}, n \geq 5$ (the degree $d$, the integer $e$ such that $\omega_{X} \simeq \mathcal{O}_{X}(e)$, the least degree, $s$, of a hypersurface containing $X$ ). In particular we prove (Theorem 3.9): $s \geq n+1$.

\section{Positivity lemma and some consequences}

Lemma 1.1 (Positivity lemma). Let $F$ be a rank two vector bundle on a smooth connected variety $X$ of dimension $m$ and let $L$ be an invertible sheaf such that $h^{0}(F \otimes L) \neq 0 . P u t$

$$
\frac{1+c_{1}(L) t}{1-c_{1}(F) t+c_{2}(F) t^{2}}=\sum u_{i} t^{i}
$$

in $A_{*}(X)[[t]]$, where $A_{*}(X)$ is the Chow ring of $X$ and $t$ is an indeterminate. Assume that $F$ is globally generated. Then the $u_{i}$ 's can be represented by pseudo-effective cycles (see [9], 2.2.B), in particular $u_{m}$ has non-negative degree.

Proof. Set $Q:=P(F)$ (in Grothendieck notation $\operatorname{Proj}(\operatorname{Sym} F)$ ) and denote by $p: Q \rightarrow X$ the projection. The Chow ring of $Q$ is

$$
\frac{A_{*}(X)[x]}{\left(x^{2}-c_{1}(F) x+c_{2}(F)\right)}
$$

(where the indeterminate $x$ corresponds to the tautological quotient of $p^{*}(F)$ ) and the Gysin map $p_{*}: A_{*}(Q) \rightarrow A_{*}(X)$ sends $\alpha+\beta x$ to $\beta$. By hypothesis, there is an effective divisor $D$ of first Chern class $x+c_{1}(L)$. Since $F$ is globally generated $x$ is nef and $D \cdot x^{i}$ is pseudo-effective. Then $p_{*}\left(D \cdot x^{i}\right)=p_{*}\left(x^{i+1}+c_{1}(L) x^{i}\right)=u_{i}$ (by the formula giving the Gysin map), so $u_{i}$ is pseudo-effective.

We will apply the lemma in the following situation:

$X$ is a subvariety of codimension two of $\mathbb{P}^{n}$ (i.e. $n=m+2$ ) and $F=N_{X}(-1)$. One knows that $F$ is globally generated because it is a quotient of $T_{\mathbb{P}^{n}}(-1)$, which is globally generated on $\mathbb{P}^{n}$. Then we will consider two cases separately:

(1) $n=5$.

(2) $\omega_{X}=\mathcal{O}_{X}(e)$ for some integer $e$ (by [2] this is always satisfied if $n \geq 6$ ). 
Recall that in the last situation we have an exact sequence

$$
0 \rightarrow \mathcal{O} \rightarrow E \rightarrow I_{X}(e+n+1) \rightarrow 0,
$$

where $E$ is a rank 2 vector bundle on $\mathbb{P}^{n}$ with Chern classes $c_{1}(E)=e+n+1$, $c_{2}(E)=\operatorname{deg}(X)$, and that $N_{X}=E \otimes \mathcal{O}_{X}$.

Lemma 1.2. Let $X \subset \mathbb{P}^{5}$ be a smooth codimension two subvariety of degree d lying on a hypersurface $\Sigma$ of degree $s$. Denote by $\pi$ the sectional genus of $X$ and assume that $X \not \subset \operatorname{Sing} \Sigma$. Then one has

$$
0 \leq \mu:=d\left(s^{2}-4 s+d\right)-s(2 \pi-2) \leq s(s-1)^{3} .
$$

Proof. The computations are made in $\operatorname{Num}(X)=A(X) /$ (numerical equivalence) (so $\operatorname{Num}^{3}(X) \simeq \mathbb{Z}$ ). We denote by $C_{i}$ the Chern classes of $N_{X}(-1)$, by $h$ (resp. $k$ ) the class of $\mathcal{O}_{X}(1)\left(\right.$ resp. $\left.\omega_{X}\right)$. Finally $y$ will denote the element $c_{2}\left(N_{X}(-s)\right) \in$ $\operatorname{Num}^{2}(X)$.

Since $X \not \subset \operatorname{Sing} \Sigma$, we have $h^{0}\left(N_{X}(s-e-n-1)\right)=h^{0}\left(N_{X}^{*}(s)\right) \neq 0$ hence we may apply Lemma 1.1 with $F=N_{X}(-1)$ and $L=\mathcal{O}_{X}(s-e-n)$ and the $u_{i}$ 's are pseudo-effective.

We have

$$
u_{2}=(s-1) h C_{1}-C_{2}=(s-1)^{2} h^{2}-y
$$

and

$$
u_{3}=(s-1) h\left(C_{1}^{2}-C_{2}\right)-C_{1} C_{2}=(s-1)^{3} h^{3}-\left((s-1) h+C_{1}\right) y .
$$

We know that $C_{1}=4 h+k$ and $y=\left(s^{2}-6 s+d\right) h^{2}-s h k$ (this follows expressing this $c_{2}$ in function of $c_{2}\left(N_{X}\right)$ which is $d h^{2}$ by the self intersection formula). The relation $u_{3} \geq 0$ is equivalent (in $\mathbb{Z}$ ) to:

$$
\begin{aligned}
0 & \leq(s-1)^{3} h^{3}-[(s+3) h+k] \cdot\left[\left(s^{2}-6 s+d\right) h^{2}-s h k\right] \\
& =-[d(s+3)-21 s+1] h^{3}-(d-9 s) h^{2} k+s h k^{2} .
\end{aligned}
$$

Let us write $h^{2} k$ as a function of $\mu$ :

$$
h^{2} k=\frac{\left(s^{2}-6 s+d\right) h^{3}-\mu}{s} .
$$

Apply "Hodge index" to the hyperplane section of $X$ : setting $\delta=\left(h^{2} k\right)^{2}-h^{3} \cdot\left(h k^{2}\right) \geq$ 0 , we get (with $d=h^{3}$ )

$$
h k^{2}=\frac{\left(h^{2} k\right)^{2}}{d}-\frac{\delta}{d}
$$


We partially eliminate $h^{2} k$ :

$0 \leq-d[d(s+3)-21 s+1]+h^{2} k\left[-(d-9 s)+\frac{s}{d}\left(\left(s^{2}-6 s+d\right) \frac{d}{s}-\frac{\mu}{s}\right)\right]-\frac{s \delta}{d}$.

This yields

$$
0 \leq-d[d(s+3)-21 s+d]+h^{2} k\left[s(s+3)-\frac{\mu}{d}\right]-\frac{s \delta}{d} .
$$

We eliminate $h^{2} k$ :

$$
0 \leq-d[d(s+3)-21 s+d]+\left[\left(s^{2}-6 s+d\right) d-\mu\right]\left(s+3-\frac{\mu}{d s}\right)-\frac{s \delta}{d},
$$

this can be written

$$
0 \leq(s-1)^{3} d-\mu\left(2 s-3+\frac{d}{s}\right)+\frac{\mu^{2}}{d s}-\frac{s \delta}{d}
$$

Now multiply by $s / d$ :

$$
0 \leq s(s-1)^{3}-\mu\left[1+\frac{s(2 s-3)}{d}\right]+\frac{\mu^{2}}{d^{2}}-\delta \frac{s^{2}}{d^{2}} .
$$

The relation $u_{2} \geq 0$ implies that $\mu \leq d(s-1)^{2}$, so,

$$
\begin{aligned}
0 & \leq s(s-1)^{3}-\mu+\frac{\mu}{d}\left[-s(2 s-3)+\frac{\mu}{d}\right]-\delta \frac{s^{2}}{d^{2}} \\
& =(s-1)^{3}-\mu+\frac{\mu}{d}\left[(s-1)^{2}-s(2 s-3)\right]-\frac{\mu}{d}\left[(s-1)^{2}-\frac{\mu}{d}\right]-\delta \frac{s^{2}}{d^{2}} .
\end{aligned}
$$

Finally

$$
0 \leq s(s-1)^{3}-\mu-\frac{\mu}{d}\left(s^{2}-s-1\right)-\frac{\mu}{d}\left[(s-1)^{2}-\frac{\mu}{d}\right]-\delta \frac{s^{2}}{d^{2}},
$$

and the lemma follows.

The last lemma will be used in Section 2.

In the second case let $s=\min \left\{t: h^{0}\left(\mathcal{I}_{X}(t) \neq 0\right\}\right.$ and $q=\min (s, e+n)$, and notice that $h^{0}\left(N_{X}^{*}(q)\right)>0$. Apply the positivity lemma with $L=\mathcal{O}_{X}(q-e-n)$. Then the $u_{i}$ 's can be computed in $A_{*}\left(\mathbb{P}^{n}\right)=\frac{\mathbb{Z}[t]}{t^{n+1}}$ (by abuse of notation we consider $u_{i}$ as an integer instead of an element of $\left.\mathbb{Z} t^{i}\right)$ and the positivity lemma applied to $X \cap \mathbb{P}^{i+2}$ says that $u_{i} \geq 0$ for $i \leq n-2$. Let $s_{i}$ be the Segre classes of $E(-1)$. One has $u_{i}=c_{1}(L) \cdot s_{i-1}+s_{i}$. If $s \geq e+n$ one has $L \simeq \mathcal{O}, u_{i}=s_{i}$; this case is not new ([8]), so we focus on the other case $(q=s)$. 
Remark 1.3. The $u_{i}$ are computed by induction on $i$ by $u_{0}=1, u_{1}=s-1$, $u_{i}=(e+n-1) u_{i-1}-(d-e-n) u_{i-2}$.

Set $z:=d-s(e+n+1)+s^{2}$, then the first $u_{i}$ 's are: $u_{2}=(s-1)^{2}-z$, $u_{3}=(s-1)^{3}-z(e+n+s-2)$ and $u_{4}=u_{2}^{2}-z(e+n-1)^{2}$.

We have the non trivial inequalities $u_{i} \geq 0,2 \leq i \leq n-2$. The first is included in Lemme 1 of [7], the second immediately implies the following theorem in the case $n=5$ (see also [5]).

Theorem 1.4 (Speciality theorem). Let $X \subset \mathbb{P}^{n}, n \geq 5$, be a smooth subvariety of codimension two with $\omega_{X} \simeq \mathcal{O}_{X}(e)$. Let $\Sigma \subset \mathbb{P}^{n}$ denote a hypersurface of degree $s$ containing $X$. If $X$ is not a complete intersection then:

(i) If $n=5: e \leq \frac{(s-1)^{3}}{4}-3-s$ and $d \leq \frac{s(s-1)\left[(s-1)^{2}-4\right]}{4}+1$.

(ii) If $n \geq 6$ : $e \leq \frac{(s-1)^{2}-n+1}{\sqrt{n-1}}-n+1$ and $d \leq \frac{s\left[(s-1)^{2}-n+1\right]}{\sqrt{n-1}}+1$.

Proof. (i) By $u_{3} \geq 0$ : $(s-1)^{3} \geq z(e+n+s-2)$. Observe that, since $X$ is not a complete intersection, $z=c_{2}(E(-e-n-1+s)$ is the degree of a codimension two subscheme which is not a complete intersection. By [12], $z \geq n-1$. It follows that $(s-1)^{3} \geq(n-1)(e+n+s-2)$, which gives the bound on $e$. By $u_{2} \geq 0$ : $d \leq s(n-1+e)+1$ and this gives the bound on $d$.

(ii) The proof is similar using $u_{4} \geq 0$ instead of $u_{3} \geq 0$.

\section{Application to the biliaison classes of codimension two subvarieties of $\mathbb{P}^{5}$}

We recall that a family $\Phi$ of coherent sheaves over an algebraic variety $S$ is limited if there exists an algebraic variety $T$ and a coherent sheaf $\mathcal{F}$ over $T \times S$ such that for any member $g$ of $\Phi$ there exists a geometric point $t \in T$ such that $g$ is isomorphic to the fiber $\mathcal{F}_{t}$ of $\mathcal{F}$ over $t$.

Theorem 2.1. Fix an integer $s>0$. The family of sheaves $\mathfrak{I}_{X, \Sigma}\left(\left[\frac{d}{s}\right]\right)$, where

- $\Sigma$ is any integral hypersurface of degree $s$ in $\mathbb{P}^{5}$,

- $d$ is any integer and $X$ is a smooth threefold of degree d lying on $\Sigma$,

is limited.

Remark 2.2. The corresponding statement for $\mathbb{P}^{n}$ is

- false for $n=3$ (for $\Sigma=\mathbb{P}^{1} \times \mathbb{P}^{1}$, a quadric in $\mathbb{P}^{3}$, one gets the sheaves $\mathcal{O}(a,-a)$ if $d$ is even),

- unknown for $n=4$,

- superseded by the speciality theorem (1.4) for $n \geq 6$. 
Since the degree of $X$ is bounded when $X \subset \operatorname{Sing} \Sigma$, the family of the sheaves $\tau_{X, \Sigma}$, with $X \subset \operatorname{Sing} \Sigma$, is clearly limited. Hence in the following we will assume $X \not \subset \operatorname{Sing} \Sigma$.

Let $C$ (resp. $S$ ) denote the intersection of $X($ resp. $\Sigma)$ with a general $\mathbb{P}^{3}$ in $\mathbb{P}^{5}$.

Lemma 2.3. The sheaves $\mathcal{I}_{C, S}\left(\left[\frac{d}{s}\right]\right)$ form a limited family.

Proof. Due to the existence of Grothendieck Quot scheme, it suffices to show that:

(1) the Hilbert polynomials of these sheaves constitute a finite set,

(2) there exists an integer $N$ depending only on $s$ such that $\mathcal{I}_{X, S}\left(\left[\frac{d}{s}\right]+N\right)$ is Castelnuovo-regular.

(1) By a direct computation we have

$$
\chi\left(\mathcal{I}_{C, S}\left(\left[\frac{d}{s}\right]\right)\right)=\left(\begin{array}{c}
s+\epsilon \\
3
\end{array}\right)-\left(\begin{array}{l}
\epsilon \\
3
\end{array}\right)-\frac{\mu}{2 s},
$$

where $\mu=d\left(s^{2}-4 s+d\right)-s(2 \pi-2), \epsilon=\frac{d}{s}-\left[\frac{d}{s}\right]$. (If $s$ divides $d$, just compare $\chi\left(\mathcal{I}_{C, S}(d / s)\right)$ with $\chi\left(\mathcal{I}_{\Gamma, S}(d / s)\right)$ where $\Gamma$ is the complete intersection of $S$ with a surface of degree $d / s)$. We conclude with Lemma 1.2.

(2) We set $\mathcal{I}_{C, S}\left(\left[\frac{d}{s}\right]\right)=: \mathcal{F}$ and notice that, for degree reasons, $\mathcal{F}(s-1) \otimes \mathcal{O}_{H}$ is Castelnuovo-regular for $H$ a general plane in $\mathbb{P}^{3}$. Also $\left(\right.$ since $h^{0}(\mathcal{F}(-1))=0$ ) we have

$$
h^{0}(\mathcal{F}(s-1)) \leq \sum_{k=0}^{s-1} h^{0}\left(\mathcal{F}(k) \otimes \mathcal{O}_{H}\right) \leq \sum_{k=0}^{s-1}(s k+1),
$$

i.e., $h^{0}(\mathcal{F}(s-1))$ is bounded uniformly in $s$. It follows that $h^{1}(\mathcal{F}(s-1))$ is bounded uniformly in $s$ (since $h^{0}$ and $\chi$ are and $h^{2}(\mathcal{F}(s-1))=0$ ), say by $M$. By a classical argument $h^{1}$ is strictly decreasing after the regularity of the general plane section ([14]) and we deduce that $h^{1}(\mathcal{F}(s-1+M))=0$, so $\mathcal{F}$ is $(s+M)$-regular.

Lemma 2.4. Let $\Phi$ be a family of sheaves on $\mathbb{P}^{n}$ with the following properties:

(1) any $\mathcal{F} \in \Phi$ is locally of depth $\geq 2$;

(2) for a general hyperplane $H \subset \mathbb{P}^{n}$ the family of the restrictions of the members of $\Phi$ is limited;

(3) $h^{0}(\mathcal{F})$ is bounded uniformly in $\mathcal{F} \in \Phi$.

Then $\Phi$ is limited.

Proof. By the second assumption we know that the set of the Hilbert polynomials of $\left.\mathcal{F}\right|_{H}(\mathcal{F} \in \Phi)$ is finite, so it will be sufficient to prove the following 
Claim. $h^{1}(\mathcal{F})$ is bounded uniformly in $\mathcal{F} \in \Phi$.

In fact, by assumption (2) we know that the $h^{i}(\mathcal{F})$ 's are bounded uniformly in $\mathcal{F} \in \Phi$ when $i \geq 2$ because of the inequality $h^{i}(\mathcal{F}) \leq\left.\sum_{k \geq 0} h^{i-1} \mathcal{F}(k)\right|_{H}$. So, from (1), (3) and our claim, it follows that $|\chi(\mathcal{F})|$ is bounded uniformly in $\mathcal{F} \in \Phi$. So the Hilbert polynomial $P_{\mathscr{F}}$ of $\mathcal{F}$ is such that $P_{\mathcal{F}}(0)$ and $\left(P_{\mathscr{F}}(x+1)-P_{\mathcal{F}}(x)\right)$ form a finite set $(\mathcal{F} \in \Phi)$, which implies that the set $\left\{P_{\mathcal{F}}: \mathcal{F} \in \Phi\right\}$ is finite. A uniform bound on the regularity of $\mathcal{F}$ is obtained exactly as in the previous lemma.

To prove the claim we look at the exact sequence

$$
H^{0}\left(\left.\mathcal{F}\right|_{H}(-k)\right) \rightarrow H^{1}(\mathcal{F}(-k-1)) \rightarrow H^{1}(\mathcal{F}(-k)) \rightarrow H^{1}\left(\left.\mathcal{F}\right|_{H}(-k)\right) .
$$

There is an integer $k_{0}$ independent of $\mathcal{F}$ so that $h^{0}\left(\left.\mathcal{F}\right|_{H}\left(-k_{0}\right)\right)=0=h^{1}\left(\left.\mathcal{F}\right|_{H}\left(-k_{0}\right)\right)$. Since $\mathcal{F}$ is locally of depth $\geq 2$ we also know $H^{1}(\mathcal{F}(-k))=0$ for $k \gg 0$, and so for $k \geq k_{0}$ by using the above exact sequence. Then we have $h^{1}(\mathcal{F}) \leq$ $\sum_{0}^{k_{0}} h^{1}\left(\left.\mathcal{F}\right|_{H}(-i)\right)$.

Proof of Theorem 2.1. From Lemma 2.3 we know that the family of sheaves $\mathcal{I}_{X, \Sigma}\left(\left.\left[\frac{d}{s}\right]\right|_{\mathbb{P}^{3}}\right)$ is limited for a general $\mathbb{P}^{3} \subset \mathbb{P}^{5}$. We conclude applying two times Lemma 2.4 .

Remark 2.5. (1) If we consider the class of ideals $\mathcal{I}_{X, \Sigma}$ (as in the theorem) modulo the equivalence relation identifying two sheaves $\mathcal{L}, \mathcal{G}$ if $\mathcal{I}$ is isomorphic to some twist of $\mathscr{g}$, we could call them "biliaison classes" (on a specified hypersurface): if $\tau_{X, \Sigma} \sim \mathcal{I}_{X^{\prime}, \Sigma^{\prime}}$ then $\Sigma=\Sigma^{\prime}$ and $X^{\prime}$ and $X$ can be linked in $\Sigma$ to the same variety. Then (roughly speaking) the theorem says that when the degree of the specified hypersurface remains bounded, the set of the corresponding biliaison classes is limited.

(2) In contrast with the case $n \geq 6$, we notice that for any $s \geq 2$ one can find ACM, non complete intersection varieties of arbitrary large degree lying on a hypersurface of degree $s$.

Corollary 2.6 (compare with [3]). The family of smooth threefold in $\mathbb{P}^{5}$ which are not of general type is limited.

Proof. According to [3] (proof of Theorem 4.3) we may restrict to the threefolds lying on a hypersurface of degree 12, so we may fix $s$. Consider the corresponding family of sheaves $\mathcal{F}=I_{X, \Sigma}\left(\left[\frac{d}{s}\right]\right)$, as in Theorem 2.1. Then $\omega_{X}$ is a quotient of $\mathscr{H o m}\left(\mathcal{I}_{X, \Sigma}, \omega_{\Sigma}\right)=\mathscr{H} \operatorname{om}\left(\mathcal{F}, \omega_{\Sigma}\left(\left[\frac{d}{s}\right]\right)\right)$. Since the family $\Phi$ is limited we can find an integer $k$ (independent of $X$ ) such that $\mathscr{H} \operatorname{om}\left(\mathcal{F}, \omega_{\Sigma}(k)\right)$ is globally generated. So if $X$ is not of general type one must have $\left[\frac{d}{s}\right]<k$, hence $d<s(k+1)$. 


\section{Application to subcanonical codimension two subvarieties of $\mathbb{P}^{n}, n \geq 5$}

Notation. We are now in case 2 of Section 1, so $X$ is the zero-locus of a rank two vector bundle $E$ of Chern classes $(e+n+1, d)$. For sake of simplicity we consider the Chern polynomial $e(X)=X^{2}-C_{1} X+C_{2}$ of $E(-1)^{*}$. Let $\Delta=C_{1}^{2}-4 C_{2}$ be its discriminant. We set $\rho=\sqrt{C_{2}}$ and write $1-C_{1} X+C_{2} X^{2}=1-2 \rho \cdot \operatorname{ch} t \cdot X+\rho^{2} X^{2}$ with the convention that $t>0$ if $\Delta>0$ and $t=i \theta, 0<\theta<\pi$, if $\Delta<0$ (in this way $\operatorname{ch} t=\cos \theta, \operatorname{sh} t=i \sin \theta)$. Then the roots of $X^{2}-C_{1} X+C_{2}$ are $b=\rho e^{t}$, $a=\rho e^{-t}$. Finally we set $\sigma=\sqrt{z}$.

If $s_{k}$ is the $k$-th Segre class of $E(-1)$, i.e.

$$
\frac{1}{1-C_{1} X+C_{2} X^{2}}=\sum_{k \geq 0} s_{k} X^{k}
$$

one deduces from $1-C_{1} X+C_{2} X^{2}=\left(1-\rho e^{t} X\right)\left(1-\rho e^{-t} X\right)$, after a partial decomposition, the formula $s_{k}=\rho^{k} \frac{\operatorname{sh}(k+1) t}{\operatorname{sh} t}$ (to be replaced by $\rho^{k}(k+1)$ if $\Delta=0$ ) and

$$
u_{k}=\rho^{k}\left[\frac{s-1}{\rho} \frac{\operatorname{sh} k t}{\operatorname{sh} t}-\frac{\operatorname{sh}(k-1) t}{\operatorname{sh} t}\right]
$$

(to be replaced by $u_{k}=\rho^{k}\left[k \frac{s-1}{\rho}-(k-1)\right]$ if $\Delta=0$ ).

Lemma 3.1. Let $f$, $g$ and $v$ be functions defined by $f(x)=\frac{\operatorname{sh}(x+1) t}{\operatorname{sh} x t}, g(x)=\frac{\sin (x+1) \theta}{\sin x \theta}$ and $v(x)=\frac{(x+1)}{x}$.

(1) If $\Delta>0$, then there exists a unique $\alpha \in] 0,+\infty[$ such that $f(\alpha)=\rho /(s-1)$.

(2) If $\Delta<0$, then there exists a unique $\alpha \in] 0, \frac{\pi}{\theta}-1[$ such that $g(\alpha)=\rho /(s-1)$.

(3) If $\Delta=0$, then there exists a unique $\alpha \geq n-3$ such that $v(\alpha)=\rho /(s-1)$.

Proof. (1) The function $f$ is strictly decreasing on $] 0,+\infty[$. Moreover, if $\Delta>0$, $\lim _{x \rightarrow+\infty} f(x)=e^{t}$. Since $\Delta>0, E$ is not stable and $2 s<e+n+1$. Since $0<z=e(s-1)$, we have $s-1<a$ hence $s-1 \leq a=\rho e^{-t}, e^{t} \leq \frac{\rho}{s-1}$. We conclude that there exists a unique $\alpha$ such that $f(\alpha)=\rho /(s-1)$.

(2) In this case is $g$ is strictly decreasing on $] 0, \frac{\pi}{\theta}-1[$ and we conclude.

(3) In this case is $v$ is strictly decreasing on $] 0,+\infty$ [ so we have a unique $\alpha \in$ ] $0,+\infty[$ such that $v(\alpha)=\rho /(s-1)$. By Lemma 1.1 (see also the end of Notation), $v(n-3) \geq \rho /(s-1)$, hence $\alpha \geq n-3$. 
Lemma 3.2. With notations as above we have:

$$
\begin{array}{rlrl}
\frac{\sigma}{\operatorname{sh} t} & =\frac{s-1}{\operatorname{sh} \alpha t}=\frac{\rho}{\operatorname{sh}(\alpha+1) t}=\frac{e+n-s}{\operatorname{sh}(\alpha+2) t} & & \text { if } \Delta>0 ; \\
\frac{\sigma}{\sin \theta} & =\frac{s-1}{\sin \alpha \theta}=\frac{\rho}{\sin (\alpha+1) \theta}=\frac{e+n-s}{\sin (\alpha+2) \theta} & & \text { if } \Delta<0 ; \\
\alpha+2 & =\frac{e+n-s}{\sigma}=\frac{\rho}{\sigma}+1=\frac{s-1}{\sigma}+2 & \text { if } \Delta=0 .
\end{array}
$$

Proof. First assume that $\Delta>0$. By definition $z=e(s-1)$. Inserting $s-1=$ $\frac{\rho \operatorname{sh} \alpha t}{\operatorname{sh}(\alpha+1) t}$, we get

$z=\rho^{2}\left[\frac{\operatorname{sh}^{2}(\alpha t)}{\operatorname{sh}^{2}(\alpha+1) t}-2 \operatorname{ch} t \cdot \frac{\operatorname{sh}(\alpha t)}{\operatorname{sh}(\alpha+1) t}+1\right]=\rho^{2}\left[\frac{\operatorname{sh}^{2}(\alpha t)}{\operatorname{sh}^{2}(\alpha+1) t}-\frac{\operatorname{sh}(\alpha-1) t}{\operatorname{sh}(\alpha+1) t}\right]$.

For the last equality check that $\operatorname{sh}(\alpha+1) t+\operatorname{sh}(\alpha-1) t=2 \operatorname{ch} t \cdot \operatorname{sh}(\alpha t)$. Finally

$$
\rho^{2}\left[\frac{\operatorname{sh}^{2}(\alpha t)}{\operatorname{sh}^{2}(\alpha+1) t}-\frac{\operatorname{sh}(\alpha-1) t}{\operatorname{sh}(\alpha+1) t}\right]=\left[\frac{\rho \operatorname{sh} t}{\operatorname{sh}(\alpha+1) t}\right]^{2} .
$$

For this check that $\operatorname{sh}^{2}(\alpha t)-\operatorname{sh}(\alpha-1) t \cdot \operatorname{sh}(\alpha+1) t=\operatorname{sh}^{2}(t)$. We conclude that

$$
\frac{\sigma}{\operatorname{sh} t}=\frac{\rho}{\operatorname{sh}(\alpha+1) t} \text {. }
$$

This proves the first three equalities. For the last one:

$$
\frac{\rho}{\operatorname{sh}(\alpha+1) t}=\frac{s-1}{\operatorname{sh} \alpha t}=\frac{2 \rho \operatorname{ch} t-(s-1)}{2 \operatorname{sh}(\alpha+1) t \operatorname{ch} t-\operatorname{sh} \alpha t} .
$$

To conclude observe that $2 \rho \operatorname{ch} t=e+n-1$ and $2 \operatorname{sh}(\alpha+1) t \operatorname{ch} t-\operatorname{sh} \alpha t=\operatorname{sh}(\alpha+2) t$.

The proof in case $\Delta<0$ is similar. If $\Delta=0$, observe that $z=e(s-1)=$ $(s-\rho-1)^{2}$, hence $\sigma=\rho-s+1$.

Remark 3.3. Observe that when $\Delta<0$ and $s=e+n$, then $\sin (\alpha+2) \theta=0$.

Proposition 3.4. Keeping notations as above, we have, for $n \geq 5$ :

$$
e+n-s \leq(n-1)^{-\frac{1}{n-4}}(s-1)^{\frac{n-2}{n-4}}
$$

and

$$
d \leq s\left[1+(n-1)^{-\frac{1}{n-4}}(s-1)^{\frac{n-2}{n-4}}\right] .
$$


Proof. First of all we assume that $\Delta>0$ and we observe that $f(t)=\log \operatorname{sh} t$ is concave. Since $\alpha t=\frac{1}{\alpha} t+\frac{\alpha-1}{\alpha}(\alpha+1) t$ we have $f(a t)=f\left(\frac{1}{\alpha} t+\frac{\alpha-1}{\alpha}(\alpha+1) t\right) \geq$ $\frac{1}{\alpha} f(t)+\frac{\alpha-1}{\alpha} f((\alpha+1) t)$. Taking the exponentials we find

$$
\operatorname{sh} \alpha t \geq(\operatorname{sh} t)^{\frac{1}{\alpha}}(\operatorname{sh}(\alpha+1) t)^{\frac{\alpha-1}{\alpha}} .
$$

Similarly, writing $\alpha t=\frac{2}{\alpha+1} t+\frac{\alpha-1}{\alpha+1}(\alpha+2) t$ and exponentiating the inequality coming from the concavity of $f(t)$ we get

$$
\operatorname{sh} \alpha t \geq(\operatorname{sh} t)^{\frac{2}{\alpha+1}}(\operatorname{sh}(\alpha+2) t)^{\frac{\alpha-1}{\alpha+1}} .
$$

By Lemma 3.2, $\frac{\sigma}{\operatorname{sh} t}=\frac{h-1}{\operatorname{sh} \alpha t}=\frac{\rho}{\operatorname{sh}(\alpha+1) t}=\frac{e+n-h}{\operatorname{sh}(\alpha+2) t}$, hence (+) gives

$$
s-1 \geq(\sigma)^{\frac{1}{\alpha}}(\rho)^{\frac{\alpha-1}{\alpha}}
$$

and $(++)$ gives

$$
s-1 \geq(\sigma)^{\frac{2}{\alpha+1}}(e+n-s)^{\frac{\alpha-1}{\alpha+1}}
$$

from which it follows that

$$
\rho \leq \sigma^{\frac{-1}{\alpha-1}}(s-1)^{\frac{\alpha}{\alpha-1}}, \quad e+n-s \leq \sigma^{\frac{-2}{\alpha-1}}(s-1)^{\frac{\alpha+1}{\alpha-1}},
$$

and finally $d=\rho^{2}+e+n \leq s\left(1+\sigma^{\frac{-2}{\alpha-1}}(s-1)^{\frac{\alpha+1}{\alpha-1}}\right)$.

In order to conclude the case $\Delta>0$ it suffices to show that $\sigma^{\frac{-2}{\alpha-1}}(s-1)^{\frac{\alpha+1}{\alpha-1}} \leq$ $(n-1)^{\frac{-1}{n-4}}(s-1)^{\frac{n-2}{n-4}}$. Since $z=\sigma^{2} \geq n-1$ we have $\frac{(s-1)^{2}}{\sigma^{2}} \leq \frac{(s-1)^{2}}{n-1}$, and hence $\sigma^{\frac{-2}{\alpha-1}}(s-1)^{\frac{\alpha+1}{\alpha-1}}=\left(\frac{(s-1)^{2}}{\sigma^{2}}\right)^{\frac{1}{\alpha-1}}(s-1) \leq\left(\frac{(s-1)^{2}}{n-1}\right)^{\frac{1}{\alpha-1}}(s-1)$ and we are done because $\alpha \geq n-3$.

The case $\Delta<0(\Delta=0)$ can be proved the same way by using $f(t)=\log \sin t$ $(f(t)=\log t)$ which is concave as well for $t \in] 0, \pi[$.

In some sense the next proposition improves Theorem 1.4, except in the case $\Delta>0$ where the bound depends on $\Delta$, hence on $e$.

Proposition 3.5. Let $X \subset \mathbb{P}^{n}, n \geq 4$, be a smooth codimension two subvariety with $\omega_{X} \simeq \mathcal{O}_{X}(e)$. If $X$ is not a complete intersection, then the following holds.

(1) If $\Delta>0$, then $d<M^{2} s^{2}+s M \sqrt{\Delta}$, where $M=\frac{n-2}{n-3}$.

(2) If $\Delta \leq 0$, then $d<M^{2} s(s-1)+s$.

Proof. (1) By Lemma 3.2

$$
\frac{\rho}{s-1}=\frac{\operatorname{sh}(\alpha+1) t}{\operatorname{sh} \alpha t}
$$


and

$$
\frac{\operatorname{sh}(\alpha+1) t}{\operatorname{sh} \alpha t} \leq \frac{\operatorname{sh}(n-2) t}{\operatorname{sh}(n-3) t}
$$

since $\alpha \geq n-3$ (Lemma 3.1). One can check that

$$
\frac{\operatorname{sh}(n-2) t}{\operatorname{sh}(n-3) t} \leq e^{t} \frac{n-2}{n-3}
$$

so

$$
\frac{\rho}{s-1} \leq e^{t} \frac{n-2}{n-3}
$$

and

$$
a=\frac{\rho}{e^{t}} \leq(s-1) \frac{n-2}{n-3} .
$$

Then we have

$$
w:=a-(s-1) \leq(s-1)\left[\frac{n-2}{n-3}-1\right]=\frac{s-1}{n-3}<\frac{s}{n-3}
$$

and

$$
d=(a+1)(b+1)=(s+w)^{2}+\sqrt{\Delta}(s+w) .
$$

Finally we get $d<\left(\frac{n-2}{n-3}\right)^{2} s^{2}+s \sqrt{\Delta} \frac{n-2}{n-3}$.

(2) First assume $\Delta<0$. By Lemma 3.2 we have $\frac{\rho}{s-1}=\frac{\sin ((\alpha+1) \theta)}{\sin ((\alpha) \theta)} \leq \frac{\alpha+1}{\alpha}$, indeed $\frac{\sin x}{x}$ is decreasing on $0<x \leq \pi$. It follows that $\rho \leq M(s-1)$. Since $\rho=\sqrt{a b}=$ $\sqrt{d-e-n}$, we get the result taking into account the inequality $s(e+n+1-s) \leq d$ $(z \geq 0)$.

The case $\Delta=0$ follows directly from $u_{k} \geq 0$ (see Remark 1.3), taking into account the inequality $s(e+n+1-s) \leq d$.

Remark 3.6. Observe the limiting $(n \rightarrow+\infty)$ case of Proposition 3.5 (1): $d \leq$ $s^{2}+s \sqrt{\Delta}$, which can occur only for $X$ a complete intersection $(a+1, b+1)$.

The aim of the remaining of the paper is to improve the bound $s \geq n-1$ of [12] (resp. $s \geq n$ if $5 \leq n \leq 6$, [4]). We will distinguish several cases according to the sign of the discriminant, $\Delta$, of $X$.

Proposition 3.7. Let $X \subset \mathbb{P}^{n}, n \geq 4$, be a smooth subvariety of codimension two. Assume that $X$ is not a complete intersection.

(1) If $\Delta \geq 0$, then $s-1 \geq(n-3) \sqrt{n-1}$ and $e \geq(2 n-4) \sqrt{n-1}-n$.

(2) If $\Delta<0$ and $e+n+1 \geq 2 s$, then $s-1 \geq \frac{2}{\pi}(n-3) \sqrt{n-1}$ and $e \geq$ $\frac{2}{\pi}(2 n-4) \sqrt{n-1}-n$. 
Proof. (1) Assume first $\Delta>0$. By Lemma 3.2 we have $\frac{s-1}{\sigma}=\frac{\operatorname{sh}(\alpha t)}{\operatorname{sh} t} \geq \alpha$. Since $\sigma \geq \sqrt{n-1}$ and $\alpha \geq n-3$, we get the result. In the same way, from Lemma 3.2, $\frac{e+\bar{n}-s}{\sigma}=\frac{\operatorname{sh}(\alpha+2)}{\operatorname{sh} t} \geq \alpha+2 \geq n-1$, hence $e+n-s \geq(n-1) \sqrt{n-1}$ and the result follows.

Assume now $\Delta=0$. We have $z=(s-a-1)^{2}$. Since $z \geq n-1$, it follows that $a+1-s \geq \sqrt{n-1}$ (note that $a+1>s$ if $X$ is not a complete intersection), so $a \geq s-1+\sqrt{n-1}$ and we get $s-1 \geq \frac{(n-3)}{(n-2)}(s-1+\sqrt{n-1})$ hence $s-1 \geq$ $(n-3) \sqrt{n-1}$. We conclude as above since $\frac{e+n-s}{\sigma}=\alpha+2 \geq n-1$.

(2) By Lemma 3.2, $\frac{e+n-s}{s-1}=\frac{\sin (\alpha+2) \theta}{\sin \alpha \theta}$. The assumption $e+n+1 \geq 2 s$ implies that $\sin (\alpha+2) \theta \geq \sin \alpha \theta$. This in turn implies $\alpha \theta<\frac{\pi}{2}$ (we have $(\alpha+2) \theta<\frac{3 \pi}{2}$, cf. Definition 3.1). By Lemma 3.2, $\frac{s-1}{\sigma}=\frac{\sin (\alpha \theta)}{\sin \theta}$. Since $\sin \alpha x / \sin x$ is decreasing, we get: $\frac{s-1}{\sigma}>\frac{1}{\sin (\pi / 2 \alpha)}$, hence $s-1>\frac{2}{\pi}(n-3) \sqrt{n-1}$. The proof for $e$ is similar using $\frac{e+n-s}{\sigma}=\frac{\sin (\alpha+2) \theta}{\sin \theta}$ of Lemma 3.2.

Lemma 3.8. Let $X \subset \mathbb{P}^{n}, n \geq 6$, be a smooth subvariety of codimension two. Assume that $\Delta<0$ and $e+n+1-2 s \leq 0$.

(i) If $n \geq 6$, then $s \geq n+2$.

(ii) If $n \geq 8$, then $s \geq 3 n / 2$.

Proof. (i) If $n \geq 6$, then $e \geq n+2$ ([8]), hence $(n+2)+n+1 \leq e+n+1 \leq 2 s$, thus $s \geq n+2$.

(ii) As above using $e \geq 2 n-1$ ([8], Corollary 3.4 (i)).

Theorem 3.9. Let $X \subset \mathbb{P}^{n}, n \geq 4$, be a smooth codimension two subvariety. Assume that $\operatorname{ch}(k)=0$. If $n<6$ assume that $X$ is subcanonical. If $h^{0}\left(\mathcal{I}_{X}(n)\right) \neq 0$, then $X$ is a complete intersection.

Proof. For the case $n=4$ we refer to [6].

If $n=5$, by [4] we may assume that $s=5$, and by [1], $e \geq 3$. From $u_{3} \geq 0$ (see Remark 1.3) we get: $z \leq \frac{(s-1)^{3}}{e+n+s-2}$, i.e. $z \leq 5$. In fact $4 \leq z \leq 5$, since $z \geq n-1$ ([12]). Arguing as in [4], Lemma 2.6, every irreducible component of $Z_{\text {red }}$ appears with multiplicity, hence $Z$ is either a multiplicity $z$ structure on a linear subspace or is contained in a cubic hypersurface. The last case is not possible ([12]). In the first case by [10] (or also [11] observing that the proof of the main theorem works in the case of a codimension two linear subspace of $\left.\mathbb{P}^{5}\right), Z$ is a complete intersection.

In the case $n=6$, we have $f(z)=\frac{\left[(s-1)^{2}-z\right]^{2}}{z} \geq(e+n-1)^{2}$ because $u_{4} \geq 0$. Since $f(z)$ is decreasing and $z \geq n-1, f(n-1) \geq(e+n-1)^{2}$, i.e. $(s-1)^{2} \geq$ $\sqrt{n-1}(e+n-1)+n-1$. By [8]: $e \geq n+2$, so $(s-1)^{2} \geq \sqrt{n-1}(2 n+1)+n-1$, but this inequality is not satisfied if $s \leq n=6$. 
Finally assume that $7 \leq n$. If either $\Delta \geq 0$, or $\Delta<0$ and $e+n+1 \geq 2 s$, we conclude with Proposition 3.7. If $\Delta<0$ and $e+n+1 \leq 2 s$ we conclude by Lemma 3.8.

We conclude the paper with a remark concerning the asymptotic behaviour (for $n$ going to infinity) of the constants introduced in this paper. As an explicit example of how our remark works we give an improvement of Theorem 3.9 (and of [12]) for $n \geq 8$ (see Corollary 3.11).

Remark 3.10. In case $\Delta<0$, we may proceed as follows. By Lemma 3.2, $s-1=$ $\rho \frac{\sin (\alpha \theta)}{\sin (\alpha+1) \theta}$, so $s-1=\delta \frac{\sin (\alpha \theta)}{\sin \theta \cdot \sin (\alpha+1) \theta}$, where $\delta=\frac{1}{2} \sqrt{-\Delta}=\rho \sin \theta, \theta(\alpha+1)<\pi$. Let us denote by $m(\alpha)$ the minimum of the function $\varphi(\theta)=\frac{\sin (\alpha \theta)}{\sin \theta \cdot \sin (\alpha+1) \theta}$ on $] 0, \frac{\pi}{\alpha+1}[$. This minimum is reached for the solution, $\beta$, of $\frac{\sin (\alpha+1) \beta}{\sin \beta}=\sqrt{\alpha+1}$ and is an increasing function of $\alpha$. So we have $s-1 \geq \delta m(\alpha) \geq \delta m(n-3) \geq \frac{\sqrt{-\Delta_{\min }(n)}}{2}$. $m(n-3)$, where $-\Delta_{\min }(n)$ is the minimal value of $-\Delta$ allowed by the Schwarzenberger conditions on $\mathbb{P}^{n}$ (see [8]). It is possible to compute an approximated value of $m_{n}:=m(n-3)$. For instance we have: $m_{5}=1,6949, m_{6}=2,2845, m_{7}=2,8203$, $m_{8}=3,3233$ (and $m_{40}=16,1647$ ). Since $-\Delta_{\min }(8)=119$, we get $s-1 \geq 19$ if $n=8$, which is better than $12=\frac{3 \cdot 8}{2}$.

Let $E$ be a rank two vector bundle on $\mathbb{P}^{n}$ with Chern classes $c_{1}, c_{2}$ (and $\Delta=$ $c_{1}^{2}-4 c_{2}$ not a square). Let $R=\mathbb{Z}[X] /\left(X^{2}-c_{1} X+c_{2}\right)$. The Schwarzenberger condition says that $\operatorname{Tr}_{\mathbb{Q} R / \mathbb{Q}}\left(\begin{array}{c}\xi+k \\ n\end{array}\right) \in \mathbb{Z}$ for $\xi=$ class of $X, k \in \mathbb{Z}$. Let $p$ be a prime number, then we have three cases:

(1) inert ( $p R$ is prime),

(2) decomposable $\left(R / p R \simeq \mathbb{F}_{p} \times \mathbb{F}_{p}\right)$,

(3) ramified $(p \mid \Delta)$.

Claim. If there exists a rank 2 vector bundle $E$ of Chern classes $\left(c_{1}, c_{2}\right)$ on $\mathbb{P}^{n}$, then for each prime $p<n$, the discriminant $\Delta=c_{1}^{2}-4 c_{2}$ is a square mod $p$ (possibly 0 ).

Proof. Assuming the contrary, we may suppose that $n-1=p$ is a prime such that $\Delta$ is not a square $\bmod p$. Let $\xi$ be a root of $X^{2}-c_{1} X+c_{2}$ in $\mathbb{F}_{p^{2}}$. Then $\operatorname{Tr}_{\mathbb{F}_{p^{2}} / \mathbb{F}_{p}}(\xi(\xi+1) \ldots(\xi+p)) \equiv-\Delta \bmod p$, since $(\xi+1) \ldots(\xi+p)=F(\xi)-\xi$ where $F$ is the Frobenius automorphism of $\mathbb{F}_{p^{2}}$ and $\operatorname{Tr}_{\mathbb{F}_{p^{2}} / \mathbb{F}_{p}}(\xi(F(\xi)-\xi)) \equiv-\Delta$. So, if $x$ is the image of $X$ in $R$, one has that $\operatorname{Tr}_{R / \mathbb{Z}}(x(x+1) \ldots(x+p))$ is not divisible by $p$ and $\operatorname{Tr}_{\mathbb{Q} R / \mathbb{Q}}\left(\begin{array}{l}x+p \\ p+1\end{array}\right)=\chi(E)$ is not an integer. Contradiction.

By [13], pp. 134-135, one knows that there exists some prime $p<n$ such that $\Delta$ is not a square $\bmod p$, when $n \geq c(\log |\Delta|)^{2}$ under Generalized Riemann Hypothesis or when $n \geq 2(|\Delta|)^{A}$ without restrictions. This means that $\left|\Delta_{\min }(n)\right| \geq e^{\sqrt{\frac{n}{c}}}$ under GRH or $\left|\Delta_{\min }(n)\right| \geq\left(\frac{n}{2}\right)^{\frac{1}{A}}$. 
Corollary 3.11. Let $X \subset \mathbb{P}^{n}, n \geq 8$, be a smooth codimension two subvariety. If $X$ is not a complete intersection, then $s \geq \frac{3 n}{2}$.

Proof. First consider the case that $\Delta<0$. If $e+n+1 \leq 2 s$, the result follows from Lemma 3.8. If $e+n+1>2 s$ and $n \geq 11$, it follows from Proposition 3.7 because $1+\frac{2}{\pi}(n-3) \sqrt{n-1} \geq \frac{3 n}{2}$. If $e+n+1>2 s$ and $8 \leq n \leq 10$, it follows from Remark 3.10 since $s \geq 20$.

If $\Delta \geq 0$, by Proposition 3.7, it is enough to check that $1+(n-3) \sqrt{n-1} \geq \frac{3 n}{2}$ if $n \geq 8$.

\section{References}

[1] E. Ballico, L. Chiantini, On smooth subcanonical varieties of codimension 2 in $P^{n}, n \geq 4$. Annali Mat. Pura Appl. 135 (1983), 99-117. Zbl 0549.14015 MR 0750529

[2] W. Barth, Transplanting cohomology classes in complex-projective space. Amer. J. Math. 92 (1970), 951-967. Zbl 0206.50001 MR 0287032

[3] R. Braun, G. Ottaviani, M. Schneider, F. O. Schreyer, Boundedness for non-general type 3 -folds in $\mathbb{P}^{5}$. In Complex analysis and geometry, Univ. Ser. Math., Plenum Press, New York 1993, 311-338. Zbl 0798.14023 MR 1211889

[4] Ph. Ellia, D. Franco, On codimension two subvarieties of $\mathbb{P}^{4}, \mathbb{P}^{5}$. J. Algebraic Geom. 11 (2002), 513-533. Zbl 1057.14063 MR 1894936

[5] Ph. Ellia, D. Franco, On smooth divisors of a projective hypersurface. Preprint, 2005; arXiv:math.AG/0406497

[6] Ph. Ellia, D. Franco, L. Gruson, On subcanonical surfaces of $\mathbb{P}^{4}$. Math. Z. 251 (2005), 257-265. Zbl 1080.14051 MR 2191026

[7] G. Ellingsrud, Ch. Peskine, Sur les surfaces lisses de $\mathbb{P}^{4}$. Invent. Math. 95 (1989), 1-11. Zbl 0676.14009 MR 0969410

[8] A. Holme, M. Schneider, A computer aided approach to codimension two subvarieties of $\mathbb{P}^{n}, n \geq 6$. J. Reine Angew. Math. 357 (1985), 205-220. Zbl 0581.14035 MR 0783542

[9] R. Lazarsfeld, Positivity in algebraic geometry, II. Ergeb. Math. Grenzgeb. 49, SpringerVerlag, Berlin 2004. Zbl 1093.14500 MR 2095472

[10] N. Manolache, Multiple structures on smooth support. Math. Nachr. 167 (1994), 157-202. Zbl 0851.14020 MR 1285312

[11] N. Manolache, Nilpotent lci structures on global complete intersections. Math. Z. 219 (1995), 403-411. Zbl 0837.14039 MR 1339713

[12] Z. Ran, On projective varieties of codimension 2, Invent. Math. 73 (1983), 333-336. Zbl 0521.14018 MR 0714095

[13] J.-P. Serre, Euvres. Collected papers. Vol. III, Springer-Verlag, 1986. Zbl 0849.01049 MR 0926691 
[14] L. Szpiro, Lectures on equations defining space curves. Tata Institute of Fundamental Research, Bombay, Lect. on Math. and Phys.: Math. 62, Springer-Verlag, Berlin, New York 1979. Zbl 0442.14010 MR 0572085

Received July 12, 2006

Philippe Ellia, Università di Ferrara, Dipartimento di Matematica, Via Machiavelli 35, 44100 Ferrara, Italy

E-mail: phe@dns.unife.it

Davide Franco, Dipartimento di Matematica e Applicazioni "R. Caccioppoli”, Università Napoli “Federico II", Ple Tecchio 80, 80125 Napoli, Italy

E-mail: davide.franco@unina.it

Laurent Gruson, Département de Mathématiques, Université de Versailles-St Quentin, Versailles, France

E-mail: gruson@math.uvsq.fr 